\title{
Roboterassistierte Rehabilitation und funktionelle Elektrostimulation: Analyse der Evidenz zum Nutzen neuer Hilfsmittel innerhalb der Schlaganfallrehabilitation
}

\author{
Roboterassistierte Rehabilitation (RAR) und funktionelle Elektrostimulation (FES) werden häufig \\ ergänzend zur herkömmlichen Rehabilitation nach Schlaganfällen eingesetzt. Der klinische \\ Zusatznutzen ist jedoch oft unklar. Vor diesem Hintergrund hat das AIHTA gemeinsam mit \\ einer AWMF Leitlinienarbeitsgruppe die derzeitige Evidenz zu diesen Hilfsmitteln überprüft. \\ Die Kooperationsstudie kam zum Schluss, dass auf Basis wissenschaftlicher Nachweise zum \\ Nutzen manche Interventionen der RAR in der klinischen Praxis eingesetzt werden können, \\ ein zusätzlicher Nutzen der FES jedoch nicht nachgewiesen werden konnte.
}

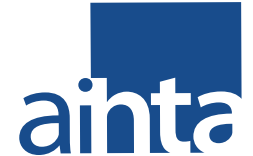

HTA Austria

Austrian Institute for Health Technology Assessment $\mathrm{GmbH}$

\section{$\mathbf{N}$}

ach Schlaganfällen leiden Betroffene häufig unter (unvollständigen) zentralen Lähmungen. Diese Lähmungen können sowohl die unteren als auch die oberen Extremitäten betreffen: RAR und FES können in diesen Fällen ergänzend zur herkömmlichen Rehabilitation eingesetzt werden. So soll die Rehabilitation von Schlaganfallpatientinnen und -patienten begünstigt werden: RAR könnte bspw. sicherstellen, dass eine intensivere und häufigere (durch Steigerung der Trainingsmotivation) Therapie ermöglicht wird, die gleichzeitig den physiotherapeutischen Aufwand reduziert. Von der FES verspricht man sich eine Stärkung des Muskels und eine verbesserte Durchblutung bzw. einen verbesserten Blutfluss.

Inwieweit diese Maßnahmen aber einen wirklichen klinischen zusätzlichen Nutzen zur herkömmlichen Rehabilitation erzielen, hat nun das AIHTA gemeinsam mit einer Arbeitsgruppe (ReMoS/Rehabilitation der Mobilität nach Schlaganfall - AG) der Arbeitsgemeinschaft der deutschen Wis- senschaftlichen Medizinischen Fachgesellschaften (AWMF) untersucht. Basis der umfassenden Analyse waren insgesamt 53 randomisierte Kontrollstudien und ein Cochrane Review. Diese Studien untersuchten den konkreten Einsatz von roboterassistierter Rehabilitation (RAR) sowie funktioneller Elektrostimulation (FES) in verschiedenen Therapiesituationen.

Die derzeitig verfügbare wissenschaftliche Evidenz deutet darauf hin, dass manche Arten der RAR (in Kombination mit der üblichen Standardrehabilitation) im Vergleich zur Standardrehabilitation allein einen klinischen Zusatznutzen bei Patienten mit Hemiparesen nach Schlaganfällen (subakute Phase) erbringen können. Eine Überlegenheit der FES (in Kombination mit der Standardrehabilitation) im Vergleich zur Standardrehabilitation konnte dagegen nicht gezeigt werden. Allerdings laufen im Moment zahlreiche Studien, die den Nutzen der RAR (24 RCTs) oder FES (6 RCTs) für die Schlaganfallrehabilitation evaluieren.
Aufgrund der großen Menge an Behandlungsoptionen, des nur begrenzt nachgewiesenen Zusatznutzens, aber der höheren Kosten mancher Interventionen sind gesundheitsökonomische Evaluationen empfehlenswert: nämlich für all jene Interventionen, die einen gewissen klinischen Nutzen oder zumindest keine Unterlegenheit vorweisen können. Hierbei sollte die (zeitliche sowie physische) Entlastung der Physiotherapeuten im Zentrum stehen.

Als unerlässlich für weitere Evaluierungen ist die Berücksichtigung der Rahmenbedingungen und des Schweregrads des Schlaganfalls zu betonen.

Goetz, G. and Walter, M. and Wohlhöfner, K. and Wittenberg, H. and Saal, S. and Stephan, K.M. and Dohle, C. (2021): Robotics and functional electrical stimulation for stroke rehabilitation. HTA-Projektbericht 128. https://eprints.ainta.at/1302/

Autor:

Gregor Goetz, MSSc, MPH

gregor.goetz@aihta.at 